\title{
Experimental Results on a Quartz Microresonator IR Sensor
}

\author{
Yoonkee Kim and John R. Vig \\ US Army Communications-Electronics Command \\ Research, Development and Engineering Center \\ Ft. Monmouth, NJ 07703
}

\begin{abstract}
The resonance frequency of a quartz bulk acoustic wave resonator is sensitive to temperature. This sensitivity has been exploited in thermometers made of single, macroscopic quartz resonators which can accurately detect temperature changes of microkelvins. It has been theoretically shown that a microresonator can be a high performance IR sensor and sensor array, combining its small thermal mass and high thermal isolation capability, the steep frequency vs. temperature characteristics of resonators made of certain cuts of quartz, and the intrinsic low noise characteristics of quartz crystal oscillators [1]. This paper is a progress report about the measurements on prototype AC-cut microresonator IR sensors operating at a $160 \mathrm{MHz}$ fundamental mode frequency. The dimensions of the microresonators were $500 \times 500 \times 10 \mu \mathrm{m}^{3}$ and $600 \times 600 \times 10 \mu \mathrm{m}^{3}$. A $10 \mathrm{~nm}$ thickness of evaporated titanium was applied to the front side of the resonators for an $\mathrm{IR}$ absorption coating. The sensors were measured in a vacuum chamber. A 500 $\mathrm{K}$ blackbody $\mathrm{IR}$ source irradiated the samples through a zinc selenide IR window. A detectivity $D^{*}$ of $8 \times 10^{7} \mathrm{cmHz}^{0.5}$ watt $^{-1}$ was achieved.
\end{abstract}

\section{INTRODUCTION}

The performance of any thermal detector is determined mainly by: 1) the steady-state response, given by:

$$
\Delta T=\frac{P}{G}
$$

where $P=$ power absorbed from the heat source, $\Delta T$ $=$ the temperature rise due to $P$, and $G=$ the thermal conductance from the sensitive element to a heat sink; 2) the noise of the detector relative to the signal produced by $\Delta \mathrm{T}$, which limits the degree to which the $\Delta \mathrm{T}$ can be resolved; and 3) the time constant $\tau_{\mathrm{T}}$ of the detector element, given by:

$$
\tau_{\mathrm{T}}=\frac{\mathrm{C}}{\mathrm{G}}
$$

where $C$ is the heat capacity of the element.

For an IR sensor, the most important parameters are the microresonators': 1) temperature coefficient of frequency, 2) noise, 3) thermal conductance to a heat sink, 4) heat capacity, and 5) IR absorbance.

Microresonators may be fabricated from a large variety of (bulk or thin film) piezoelectric materials, especially since the materials need not possess temperature-compensated cuts. Although materials other than quartz (e.g., $\mathrm{ZnO}, \mathrm{GaAs}$, langasite, etc.) may, eventually, prove to be more suitable than quartz for microresonator sensors, the discussion that follows shall be confined to quartz microresonators.

The resonator cut for microresonator sensors may be chosen from among a wide variety. Some of the possibilities are the: AC-cut $\left(20 \mathrm{ppm} /{ }^{\circ} \mathrm{C}\right.$ [2]), LC-cut $\left(35.4 \mathrm{ppm} /{ }^{\circ} \mathrm{C}\right)[3], Y$-cut $\left(\sim 90 \mathrm{ppm} /{ }^{\circ} \mathrm{C}[2]\right)$, SC-cut (b-mode: $-25.5 \mathrm{ppm} /{ }^{\circ} \mathrm{C}$ [4], or dual-mode: 80 $\mathrm{ppm} /{ }^{\circ} \mathrm{C}$ to $\left.>100 \mathrm{ppm} /{ }^{\circ} \mathrm{C}[5,6]\right)$, NLSC-cut $(\sim 14$ $\mathrm{ppm} /{ }^{\circ} \mathrm{C}$ ) [7], or any other cut that can be made thin and have a well-behaved temperature sensitive mode. A $160 \mathrm{MHz}$ AC-cut resonator was selected for the experiments primarily because of the availability of a source for fabricating such resonators [8].

The feasibility of a quartz microresonator IR sensor was theoretically discussed in a previous article [1]. An IR sensor was proposed which can be fabricated by micromachining a quartz microresonator, i.e., a high frequency resonator having etched isolation channels along its boundary.

In this paper, the results of experiments on a prototype microresonator sensor are reported. 
Experimental results of IR quartz resonator sensors have been reported previously by several authors [911]. A $30 \mathrm{MHz}$ fundamental mode $Y$-cut resonator [9] and a $10 \mathrm{MHz} 3^{\text {rd }}$ overtone SC-cut resonator [10] were employed, but the dimensions of those resonators were not in the category of a microresonator, resulting in impractically long time constants. A $100 \mathrm{MHz}$ fundamental mode Z-cut [11] was also tried; however, practical device performance was not achieved.

Quartz itself is an IR absorbing material [12], and the IR absorption of quartz resonators has been explored, e.g., by J. R. Vig for fast oscillator warmup using IR heating [13]. In order to utilize the direct absorption, the unelectroded surface area of the quartz plate needs to be maximized since the electrodes of a conventional resonator reflect most of the IR energy. Thin films that have a sheet resistance which is half the impedance of free space, i.e., $188 \Omega$ per square, can absorb $50 \%$ of incident IR radiation [14]. (The impedance of free space is $377 \Omega$.) At such an optimum film thickness, $50 \%$ of the incident IR radiation is absorbed, $25 \%$ is reflected, and $25 \%$ is transmitted. The absorption can be increased to $>50 \%$ by creating multiple passes of the IR through the front of the enclosure and a reflecting film on the resonator, e.g., by having an antireflection film on the back side. This uniform absorption coating can be applied without difficulty from $0.2 \mu \mathrm{m}$ to $8 \mu \mathrm{m}$, where strong reflection bands don't exist. In addition to the coating, ring electrodes [10] and grid electrodes [11] can also be employed.

Design goals of our resonator included a high $Q$ together with high IR absorption. Since the microresonator is to be thin (in this experiment, about $10 \mu \mathrm{m}$ ), a very thin and light $\mathrm{IR}$ absorbing coating is more desirable than a thick heavy coating. The coating should not degrade the $Q$.

\section{FABRICATION OF DEVICES}

Fig. 1 shows a photograph of a microresonator used in these experiments. It is mounted in an open $3.9 \times$ $8.4 \times 2 \mathrm{~mm}^{3}$ ceramic package. The AC-cut $\left(31^{\circ} 46^{\prime}\right.$ rotated $y$-cut) was selected because the $\mathrm{AC}$-cut has a frequency vs. temperature coefficient of +20 $\mathrm{ppm}^{\circ} \mathrm{C}$, and because a source for fabricating small, high frequency $\mathrm{AC}$-cut resonators was available [8]. The dimensions of the resonators were $400 \times 400 \times$
$10 \mu \mathrm{m}^{3}, 500 \times 500 \times 10 \mu \mathrm{m}^{3}$, and $600 \times 600 \times 10$ $\mu \mathrm{m}^{3}$. Several different sizes and shapes of electrodes were tried. The $400 \times 400 \mu^{2}$ devices showed lower Q's and were noisier than the other sizes probably because of a lack of suitable energy trapping, so the least noisy devices (at room temperature) from the $500 \times 500 \mu \mathrm{m}^{2}$ and the $600 \times$ $600 \mu \mathrm{m}^{2}$ sizes were selected for evaluation.

In the thermal IR sensor family, the thermal isolation of the sensor from the rest of the structure is one of the crucial performance issues. The isolation was accomplished with two chemically etched bridges supporting the microresonator as shown in Fig. 1. The width, thickness, and length of the bridge were designed to be $50-100 \mu \mathrm{m}, 10 \mu \mathrm{m}$, and $200-300 \mu \mathrm{m}$, respectively. The supporting frame was thick enough to provide sufficient mechanical strength for handling.

A series of IR absorption coating experiments was conducted before specifying the IR absorption coating to be used on the microresonators. Titanium and aluminum were selected, considering that their mass densities and acoustic impedances are comparable to that of quartz. Their mass density ratios to quartz are 1.8 and 0.98 , respectively. The thicknesses of $50,100,150$, and $200 \AA$ (as measured by a quartz resonator thickness monitor during evaporation) were evaporated on the IR illuminated side of $280 \mu \mathrm{m}$ thick polished AT-cut quartz plates using an e-beam evaporator. The coatings' transmission and reflection coefficients were

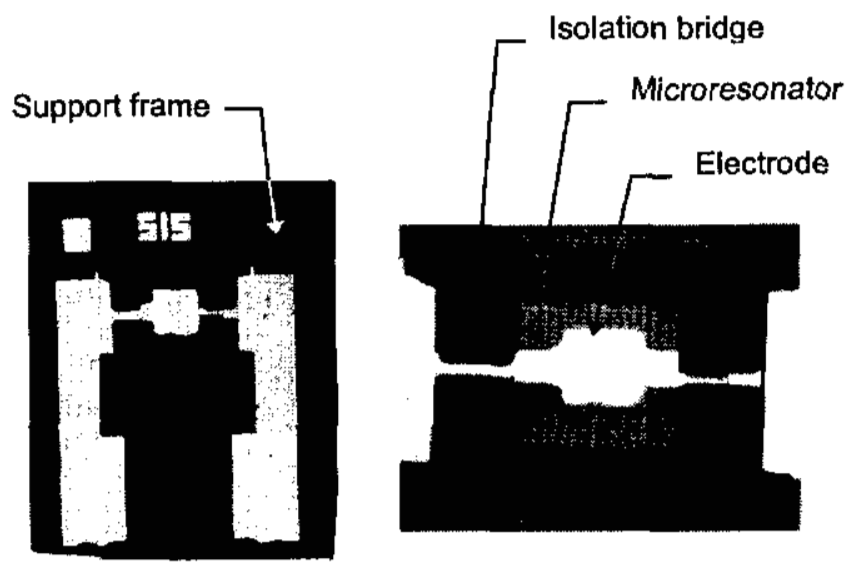

Fig. 1: Overview and close-up of $160 \mathrm{MHz}$ microresonator IR detector. 
measured with an FTIR spectrometer. The blackbody IR absorption of the bare quartz plate for $500 \mathrm{~K}$ blackbody was calculated to be $41 \%$ over the 2.5-25 $\mu \mathrm{m}$ range. Of the coatings tried, the $100 \AA$ titanium coating produced the largest increase in the IR absorption, to $51 \%$; thus, this coating was specified to be used on the $160 \mathrm{MHz}$ resonators of this experiment. The cut and thickness of quartz used in the IR absorption experiments were solely for convenience and mechanical stability. The difference in rotation angles between the AT-cut and $\mathrm{AC}$-cut does not make much difference in the overall absorption. The two cuts have slightly different spectral responses due to the anisotropy of the optical indices of quartz. The IR absorption of quartz is known to be highly anisotropic, with several absorption bands at $9,13,20$, and $26 \mu \mathrm{m}$ along reflection peaks in the near to far IR range [12]. The spectral absorption of the coatings tried did not show a uniform absorption over the wavelengths, due to these bands. A conventional gold black coating would be inappropriate for a uniform absorption spectral coating since it would imply heavy mass loading on the microresonator. Thin and light black coatings need to be developed in future experiments.

\section{MEASUREMENT METHOD}

Fig. 2 shows a block diagram of the measurement setup. The resonator under testing was placed into a vacuum chamber. The zinc selenide IR widow had an IR transmittance of $70 \%$. A vacuum level of $\sim 10^{-5}$ Torr was maintained in the chamber during the measurement to eliminate the effects of air on the $Q$ and thermal conductance. $Q$ factor measurement was not attempted in this experiment. The $500 \mathrm{~K}$ blackbody IR source with a $0.5 \mathrm{~cm}$ or $1.0 \mathrm{~cm}(0.2$ inch or $0.4 \mathrm{inch}$ ) aperture was placed $94 \mathrm{~cm}$ (37 inches) from the device.

To remove the drift of the resonant frequency due to the IR absorption caused heating, a mechanical chopper was employed to modulate the IR flux. The chopping rate employed was $1-4 \mathrm{~Hz}$, with $50 \%$ duty cycle. The chopped IR flux at the position of the device was calculated to be 0.79 and $32 \mu \mathrm{W} / \mathrm{cm}^{-2}$ for the $0.5 \mathrm{~cm}$ or $1.0 \mathrm{~cm}$ apertures, respectively.

The heat capacity and the mass of the $500 \times 500$ $\mu \mathrm{m}^{2}$ device were calculated to be $5.2 \mathrm{~J} / \mathrm{K}$ and $6.6 \mu \mathrm{g}$, respectively. The corresponding values for the $600 \times$ $600 \mu \mathrm{m}^{2}$ device were $7.5 \mathrm{~J} / \mathrm{K}$ and $9.5 \mu \mathrm{g}$. The thermal time constants for both sizes were 100-170 $\mathrm{ms}$, calculated from the dimensions of the isolation bridges and of the microresonator.

A network analyzer was utilized to track the resonant frequency. To follow the frequency modulated by the chopping was not possible in a frequency scanning mode since tracing the resonant frequency was slow compared to the response of the sensor. Thus, the measurement was performed with a fixed frequency measurement of $\$ 11$. The fixed frequency was picked at the resonant frequency from the minimum S11 data point at the onset of the measurement using a control program. The measurement bandwidth was $10 \mathrm{~Hz}$ (the minimum bandwidth of the network analyzer) and 1024 data points with $0.1 \mathrm{~s}$ interval (i.e., a $102 \mathrm{~s}$ period) were collected by the controller.

\section{RESULTS}

The magnitude and phase data were analyzed with $2^{\mathrm{N}}$ points (where $\mathrm{N}$ is an integer) Fourier transform in complex domain to reveal the frequency component associated with the chopping frequency. At least 256 data points (i.e., a $25.6 \mathrm{~s}$ period) were needed to reveal a conspicuous chopping frequency peak from the background. Further digital filtering was not applied here. Real domain Fourier transform was also applied to the magnitude and phase. The peak was more conspicuous in the phase

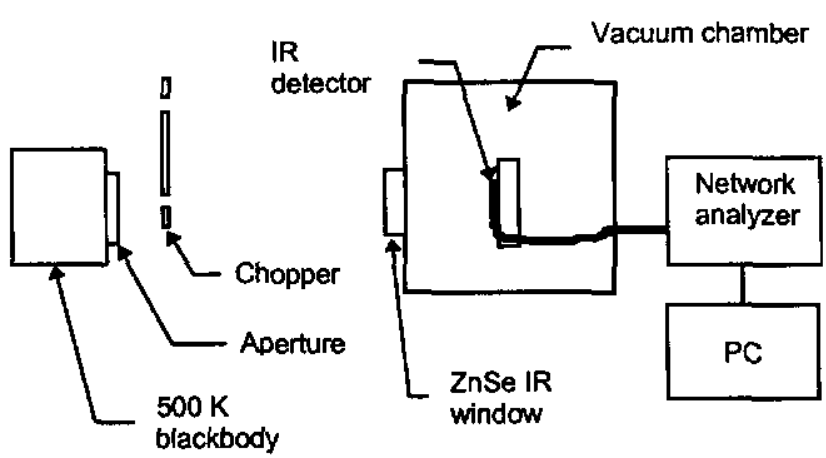

Fig. 2: Schematic of IR measurement setup. 
than the magnitude due to higher sensitivity of the phase measurement. The real domain Fourier transform applied to the phase data could give similar results with the complex domain Fourier transform to the phase and the magnitude data.

A typical magnitude versus Fourier frequency of the complex domain Fourier transformed data is shown in Fig. 3. The scale in the magnitude axis is in an arbitrary unit. The steady rise of the magnitude toward the DC component is caused by the drift of the resonant frequency due to heating by the absorbed IR. The peak around $1.5 \mathrm{~Hz}$ associated with the chopping frequency can be easily recognized. The peak could be observed up to a 4 $\mathrm{Hz}$ chopping rate, with decreased magnitude as the chopping rate approaches the time constant of the sensor. At the fast chopping rate, the chopped IR flux would be like a constant flow of an averaged IR flux, resulting in a constant shift of the resonant frequency. This shift could be measured with other measurement implementation, e.g., by measuring the beat frequency between an IR sensor and another resonator which is shielded from the IR radiation.

A rough estimate of the short term stability, $\sigma_{\mathrm{Y}}(\tau)$, at $\tau=1 \mathrm{~s}$ and at room temperature, was about $3 \times 10^{-8}$, which was equivalent to $1.5 \mathrm{mK}$ (or $8 \mathrm{~nJ}$ of thermal energy) detectability. This included resonator noise and the fluctuations in ambient temperature. (The best resonator noise floor achievable at $160 \mathrm{MHz}$ is about $2 \times 10^{-12}$, so there is plenty of room for improvement.)

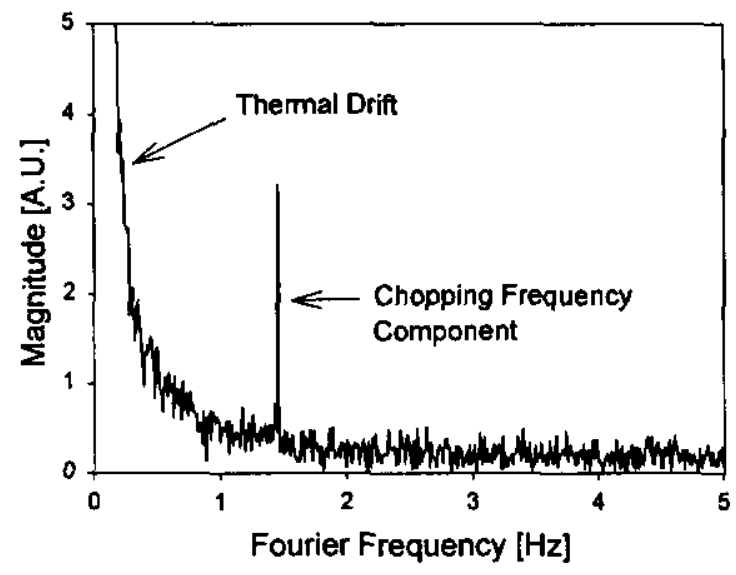

Fig. 3: Typical 1024 point Fourier transformed data.
In this experiment, when the chopping frequency component is visible from the Fourier transformed data, the microresonator is regarded as being capable of detecting the incident IR flux. This can be translated into a figure of merit for IR sensors, detectivity $\mathrm{D}^{*}$. Noise equivalent power $P_{N}$, which is the amount of incident rms signal power having an output signal-to-noise ratio of unity, is obtained by

$$
P_{N}=H \cdot A_{D}
$$

where $H$ is the irradiance and $A_{D}$ is the sensor area. The definition of $\mathrm{D}^{*}$ is [15]

$$
D^{*}=\frac{\sqrt{A_{D} \cdot B W}}{P_{N}}
$$

where $B W$ is the measurement bandwidth.

The $D^{*}$ of the prototype microresonator IR sensor was calculated to be $8 \times 10^{7} \mathrm{cmHz}^{0.5}$ watt $^{-1}$. This can be compared with the previously reported quartz resonator $\mathrm{D}^{*}, 1.3 \times 10^{6} \mathrm{cmHz}^{0.5}$ watt $^{-1}$ [11]. However, the $D^{*}$ is not yet as high as those of the best IR detectors; e.g., thermopiles, bolometers, and pyroelectric sensors exhibit $D^{*}$ 's of $<5 \times 10^{9}$, < $5 \times 10^{9}$, and $\leq 8 \times 10^{8} \mathrm{cmHz}^{0.5}$ watt $^{-1}$, respectively [16]. By developing a higher $Q$, lower noise resonator and measurement system, and with a better absorption coating than the prototype, $D^{*}$ greater than $10^{9}$ $\mathrm{cmHz}^{0.5}$ watt $^{-1}$ ought to be possible [1]. Further measurements will be necessary to determine the IR spectral response of our device.

\section{SUMMARY}

Several prototypes of a $160 \mathrm{MHz}$ AC-cut quartz microresonator IR sensor were fabricated and analyzed. The IR absorbing coating was $10 \mathrm{~nm}$ of evaporated titanium. The blackbody detectivity $\mathrm{D}^{*}$ of $8 \times 10^{7} \mathrm{cmHz}^{0.5}$ watt $^{-1}$ was measured vs. the previously reported results for a quartz resonator of $1.3 \times 10^{6} \mathrm{cmHz}^{0.5} \mathrm{watt}^{-1}$. The time constants of the devices were calculated to be $100-170 \mathrm{~ms}$. Although the performance does not yet match that of the best commercially available IR detectors (which employ different technologies), calculations based on the known properties of quartz resonators and 
oscillators predict that $\mathrm{D}^{*}$ on the order of $10^{9}$ $\mathrm{cmHz}^{0.5}$ watt $^{-1}$ ought to be achievable. Higher $\mathrm{Q}$, lower noise resonators and measurement systems, as well as better IR absorption coating, will need to be developed before such results can be achieved.

With the development of appropriate coatings, other applications of the microresonators can be in physical, chemical, and biological sensors [17].

\section{ACKNOWLEDGMENTS}

The authors would like to thank Ms. S. Laffey for building the vacuum chamber and providing the IR coatings, and Prof. D. Pierce of William Paterson Univ., NJ for his comments. The microresonators were fabricated by Statek Co. at Orange, CA.

\section{REFERENCES}

[1] J. R. Vig, R. L. Filler, and Y. Kim, "Uncooled IR imaging array based on quartz microresonators," IEEE J. Microelectromechanical Systems, Vol. 5, No. 2, pp. 131$137,1996$.

[2] R. A. Heising, Quartz Crystals for Electrical Circuits, pp. 26-27, 32, D. Van Nostrand Co., 1946.

[3] D. L. Hammond, C. A. Adams and P. Schmidt, "A Linear, Quartz Crystal, Temperature Sensing Element," ISA Transactions, Vol. 4, pp. 349-354, 1965.

[4] J. A. Kusters, M. C. Fisher, and J. G. Leach, "Dual Mode Operation of Temperature and Stress Compensated Crystals," Proc. 32nd Ann. Symp. on Frequency Control, pp. 389-397, 1978.

[5] S. S. Schodowski, "Resonator Self-Temperature Sensing Using a Dual-Harmonic-Mode Crystal Oscillator," Proc. 43rd Ann. Symp. on Frequency Control, pp. 2-7, 1989.

[6] R. L. Filler and J. R. Vig, "Resonators for the Microcomputer Compensated Crystal Oscillator," Proc. 43rd Ann. Symp. on Frequency Control, pp. 8-15, 1989.

[7] M. Nakazawa, H. Yamaguchi, A.D. Ballato, and T.J. Lukaszek, "Stress-Compensated Quartz Resonators Having Ultralinear Frequency-Temperature Responses," Proc. 38th Ann. Frequency Control Symp., pp. 240-244, 1984.

[8] Statek Co., Orange, CA.

[9] H. Ziegler and J. Tiesmeyer, "Digital Sensor for IR Radiation," Sensors and Actuators, Vol. 4, pp. 363-367, 1983.

[10] M. R. Hamrour and S. Galliou, "Analysis of the infrared sensitivity of a quartz resonator application as a thermal sensor," Proc. Ultrasonics Symp., pp. 513-516, 1994.

[11] J. E. Ralph, R. C. King, J. E. Curran and J. S. Page, "Miniature Quartz Resonator Thermal Detector," Proc. 1985 Ultrasonics Symp., pp. 362-364, 1985.
[12] W. G. Spitzer and D. A. Kleiman, "Infrared lattice bands of quartz," Physical Review, Vol. 121, No. 5, pp. 1324-1335, 1961.

[13] J. R. Vig, "Fast warm-up oven controlled piezoelectric oscillator," U.S. Patent No. 4,259,606.

[14] L. N. Hadley and D. M. Dennison, "Reflection and Transmission Interference Filters," J. Opt. Soc. Am., Vol. 37, pp. 451-465, 1947.

[15] P. W. Kruse, L. D. McGlauchin, and R. B. McQuistan, Elements of infrared technology: generation, transmission, and detection, John Wiley \& Sons, Inc., NY, 1963.

[16] K. J. Stahl, "Infrared detectors: Expanded applications prompt developments in technology," in Photonics design \& applications handbook, 41" Ed., Laurin Publishing Co., Pittsfield, MA, p. H-134, 1995.

[17] J. R. Vig, R. L. Filler, and Y. Kim, "Chemical sensor based on quartz microresonators," IEEE J. Microelectromechanical Systems, Vol. 5, No. 2, pp. 138-140, 1996. 